\title{
Dificuldades encontradas pela equipe de enfermagem no atendimento pré-hospitalar
}

\author{
Difficulties encountered by nursing team in prehospital care
}

\section{Dificultades encontradas por el equipo de enfermería en la atención prehospitalaria}

\begin{abstract}
Adson Pereira dos Santos ${ }^{1}$, Ricardo Bruno Santos Ferreira ${ }^{1 *}$, Elaine de Oliveira Souza Fonseca $^{1}$, Claudia Franco Guimarães ${ }^{1}$, Lorena Rodrigues de Carvalho ${ }^{1}$, Rafaella Fernandes Oliveira $^{1}$, Eliardo da Silva Oliveira ${ }^{1}$.
\end{abstract}

\section{RESUMO}

Objetivo: Compreender as dificuldades encontradas pela equipe de enfermagem durante o Atendimento PréHospitalar. Métodos: Estudo descritivo, exploratório com abordagem qualitativa desenvolvido em um município do Território Sertão Produtivo da Bahia, no período de dezembro de 2018, com 14 profissionais de enfermagem. Como ferramenta de coleta de dados foi utilizado uma entrevista norteada por um roteiro semiestruturado contendo informações relacionadas ao objeto do estudo. Os dados foram sistematizados através da análise de conteúdo em suas três etapas: pré análise, exploração do material e tratamento dos dados. Resultados: Evidenciou-se inúmeros entraves para o atendimento enfermagem no ambiente pré-hospitalar com destaque para a acessibilidade aos locais de atendimento, segurança da cena e a ocorrência de violência ocupacional contra os profissionais. Para superação destas dificuldades, mostrou-se como fundamental a boa comunicação com a comunidade e a existência de educação permanente com a equipe e população. Conclusão: Para tornar o atendimento mais eficaz é necessário a construção de abordagens que perpassem horizontalmente os cenário micro e macroorganizacional, onde comunidade, profissionais e gestão são corresponsáveis pelo serviço prestado.

Palavras-chave: Assistência pré-hospitalar, Socorro de urgência, Ambulâncias, Cuidados de enfermagem.

\begin{abstract}
Objective: Understand the difficulties encountered by nursing staff during the Prehospital Care. Methods: A descriptive and exploratory study with qualitative approach developed in a municipality Land Hinterland Production of Bahia, from December 2018, with 14 nursing professionals. Data collection tool was used one guided by a semi-structured interview containing information related to the object of study. The data were organized by content analysis in its three stages: pre analysis, material exploration and processing of data. Results: It was evidenced numerous barriers to nursing care in the prehospital environment for highlighting the accessibility of care facilities, security scene and the occurrence of occupational violence against professionals. To overcome these difficulties, it was shown how important good communication with the community and the existence of lifelong learning with staff and population. Conclusion: To make the service more effective is necessary to build approaches that cut across horizontally micro and macro-organizational setting where community, professional and management are co-responsible for the service.
\end{abstract}

Key words: Prehospital care, Emergency relief, Ambulances, Nursing care.

\section{RESUMEN}

Objetivo: Entender las dificultades encontradas por el personal de enfermería durante la atención prehospitalaria. Métodos: Estudio descriptivo y estudio exploratorio con enfoque cualitativo desarrollado en un municipio Tierra Hinterland Producción de Bahía, de diciembre de 2018, con 14 profesionales de enfermería. herramienta de recolección de datos se utilizó un guiado por una entrevista que contiene información semiestructurada relacionado con el objeto de estudio. Los datos fueron organizados por análisis de contenido en sus tres etapas: análisis pre, exploración de material y procesamiento de datos. Resultados: Se evidenció numerosas barreras para la atención de enfermería en el entorno prehospitalario para poner de relieve la accesibilidad de los servicios de atención, escena de la seguridad y la ocurrencia de violencia laboral contra los profesionales. Para superar estas dificultades, se ha demostrado la importancia de la comunicación buena con la comunidad y la existencia de aprendizaje permanente con el personal y la población. Conclusiones: Para hacer el servicio más eficaz es necesaria la construcción de planteamientos que engloben ajuste horizontal micro y macro-organización en la comunidad, profesional y de gestión son co-responsables del servicio.

Palabras clave: Atención pre hospitalaria, Socorro de urgência, Ambulancias, Atención de enfermería.

'Universidade do Estado da Bahia (UNEB), Guanambi - BA. *E-mail: ricardobrunoenf@gmail.com

SUBMETIDO EM: 4/2020 | ACEITO EM: 5/2020 | PUBLICADO EM: 7/2020 


\section{INTRODUÇÃO}

O atendimento pré-hospitalar (APH) refere-se à modalidade de serviço de saúde responsável por prestar o atendimento primário ao paciente após ter sofrido algum agravo à saúde, de cunho clínico ou traumático. Sendo assim, a assistência busca realizar as manobras necessárias para estabilizar o quadro apresentado (BRASIL, 2012).

No Brasil, o Serviço de Atendimento Móvel de Urgência (SAMU) teve sua implantação em 2004 por meio do Decreto ํㅜ 5.055, de 27 de abril de 2004. Segundo o Departamento de Informática do SUS (DATASUS), o serviço foi implantado seguindo o modelo francês Service d'AideMédicale d'Urgence. Foi idealizado em 1986, sendo considerado por muitos especialistas como o melhor serviço do mundo nesta modalidade (BRASIL, 2012).

O serviço do SAMU é dividido em Suporte Básico de Vida (SBV) e Suporte Avançado de Vida (SAV), onde a assistência é realizada por uma equipe multiprofissional. A equipe do SBV é formada por um técnico de enfermagem e um condutor socorrista, enquanto o SAV é composto pelo médico, enfermeiro e condutor. A inserção da enfermagem na equipe do SAMU se estende desde a assistência direta às vítimas até os cargos de gestão e responsabilidade técnica. Além disso, a enfermagem, está presente em todas as categorias que o serviço dispõe, sejam elas de suporte terrestre, marítimo ou aéreo (LUCHTEMBERG MN e PIRES DEP, 2015).

$\mathrm{O} A P H$ é realizado através de uma série de procedimentos que promovem atendimento a vítima, visando a resolutividade de situações de urgência e emergência. Para tanto, ainda que não haja grande aporte tecnológico para a execução do serviço, os profissionais envolvidos vão para a cena munidos de todos os insumos necessários para a prestação do serviço (LIMA ALP, et al., 2017).

Segundo o Pre hospital Trauma Life Support (PHTLS) (2017) o atendimendo prestado pelos socorristas em situações traumáticas é determinante para evolução do quadro dos pacientes, uma vez que este atendimento primário irá influenciar nas condições em que o paciente será encaminhado para o serviço de referência. Ademais, é através das avaliações adotadas durante a assistência que será possível previnir e detectar a existência e nível de sequelas das vítimas.

Por tratar-se de um ambiente imprevisível, os socorristas devem possuir conhecimento teórico-prático uma vez que o atendimento deve ser rápido, coordenado e eficaz. Acrescenta-se ainda a necessidade do profissional possuir preparo físico e psicológico para atuar nas diversas situações emergenciais, dada as possíveis adversidades (LUCHTEMBERG LM e PIRES DEP, 2015).

No que tange o profissional de enfermagem, a compreensão acerca dos mecanismos que permeiam a sua atuação no APH é pouco conhecida, uma vez que este serviço é pouco difundido na grade curricular da graduação em Enfermagem.

Ademais, evidenciou-se pouca produção de científica com abordagem voltada para os desafios enfrentados pela equipe de APH. Em pesquisa realizada na Biblioteca Virtual da Saúde (BVS) utilizando os descritores combinados "enfermagem em emergência", "serviços médicos de emergência", "socorro de urgência" separados pelo operador booleano "and', foram encontrados 138 artigos, dos quais apenas um se relacionava diretamente com o objeto estudado.

Diante disso, o presente estudo foi estruturado a partir da seguinte questão norteadora: Quais as dificuldades enfrentadas pela equipe de enfermagem no atendimento pré-hospitalar para a prestação de socorro às vítimas?

Acredita-se que a temática contribui para o debate acerca das barreiras existentes para execução do trabalho em enfermagem nesta modalidade de atendimento. Conhecer tais dificuldades torna-se importante para que se possa traçar estratégias de melhorias dos serviços, discutindo com os profissionais formas de aprimoramento da assistência no ambiente extra hospitalar.

A partir disso, delimitou-se como objetivo estudo compreender as dificuldades encontradas pela equipe de enfermagem durante o Atendimento Pré-Hospitalar. 


\section{MÉTODOS}

Trata-se de um estudo descritivo, exploratório, com abordagem qualitativa, realizado na base regional do Serviço de Atendimento Móvel de Urgência (SAMU) de um município de médio porte, referência do Território Sertão Produtivo da Bahia, com 14 profissionais da equipe de enfermagem, sendo 9 enfermeiros e 5 técnicos de enfermagem (MINAYO MCS, 2010).

Foram incluídos no estudo: enfermeiros e técnicos de enfermagem, de ambos os sexos, com vínculo empregatício no município e, com idade superior a 18 anos. Por outro lado, foram excluídos os profissionais que estavam afastados do serviço por motivo de férias e/ou licenças, bem como profissionais de outras categorias profissionais.

Os participantes foram abordados de forma individualizada, para que fossem mantidos a confidencialidade e anonimato, com apresentação da proposta e objetivo do estudo, seguido da apresentação e leitura do Termo de Consentimento Livre e Esclarecido (TCLE). Após aceite e assinatura do TCLE, os profissionais foram encaminhados para sala de reuniões da sede do SAMU, a fim de evitar barulhos excessivos ou interrupções, além de garantir a privacidade do entrevistado.

Os dados foram coletados no período de dezembro de 2018 através de uma entrevista norteada por um roteiro de pesquisa semiestruturado. O instrumento contém informações relacionadas aos dados sociodemográficos do participante, além de informações referentes ao objetivo proposto. Para realização das entrevistas foi utilizado um aparelho celular na função gravador de voz, salvo em mídia formato MP3. Os dados sociodemograficos foram organizados com a utilização do Microsoft Office Excel, versão 2016.

As entrevistas foram transcritas na íntegra no Microssoft Office Word e sistematizadas através da técnica de análise de conteúdo de Bardin, observando os pressupostos: 1) pré-análise, através da leitura flutuante do material transcrito; 2) exploração do material, a partir da codificação, classificação e categorização e; 3) tratamento dos resultados, balizado na literatura existente.

Após a pré-análise, os dados foram organizados de acordo a similaridade, divergência e complementariedade das informações. Durante a exploração do material emergiram duas categorias para análise: 1) Entraves para a assistência de enfermagem no ambiente pré-hospitalar; 2) A educação com instrumento de melhoria da assistência prestada no atendimento pré-hospitalar.

O estudo obedeceu às normas da Resolução n466 de 2012 e 510/2016 do Conselho Nacional de Saúde que regulamenta as pesquisas com seres humanos. Nesta perspectiva, o estudo foi aprovado pelo Comitê de Ética em Pesquisa da Universidade do Estado da Bahia sob número de CAAE: 93920718.0.0000.0057.

\section{RESULTADOS E DISCUSSÃO}

No que se refere a caracterização dos participantes, o público foi composto majoritariamente por pessoas do sexo masculino (57,14\%), com faixa etária predominante de 30 a 39 anos de idade (50\%) e pertencente à raça/cor negra $(57,14 \%)$.

Com relação à ocupação exercida pelos profissionais, $64,29 \%$ são enfermeiros e $35,71 \%$ são técnicos de enfermagem. Do total, $78,57 \%$ desses profissionais trabalham na instituição há um período de 0 a 5 anos. Os dados sociodemográficos estão apresentados na Tabela 1, apresentada mais abaixo.

A partir do perfil dos participantes nota-se uma maior inserção de pessoas do sexo masculino na profissão. A enfermagem é reconhecida historicamente por ser uma profissão feminina, no entanto, nos últimos anos a presença masculina vem crescendo, sobretudo nos setores de $\mathrm{APH}$, no qual exige maior esforço físico durante os atendimentos (MACHADO MH, et al., 2015; SANTANA JCB, et al., 2015).

A baixa faixa etária dos profissionais corrobora com outros estudos que apontam tratar-se de uma profissão que se encontra em pleno rejuvenescimento, com uma média de idade de 36 a 50 anos (SANTANA JCB, et al., 2015). Por outro lado, a presença de jovens nos setores de urgência, predispõe o aumento dos níveis de estresse uma vez que exige o desenvolvimento de autonomia e responsabilidade (MACHADO MH, et al., 2015). 
Quanto a raça/cor e estado civil dos entrevistados, verifica-se um perfil similar aos achados de Machado e colaboradores quando traçaram o perfil sociodemográfico dos profissionais de enfermagem do âmbito nacional, e observou predominância de indivíduos casados e que se autodeclaram como pardos ou pretos (SANTANA JCB, et al., 2015).

\begin{tabular}{|c|c|c|}
\hline Variável & $\mathbf{N}$ & $\%$ \\
\hline \multicolumn{3}{|l|}{ Sexo } \\
\hline Masculino & 8 & 57,1 \\
\hline Feminino & 6 & 42,9 \\
\hline \multicolumn{3}{|l|}{ Idade } \\
\hline 20 a 29 & 3 & 21,4 \\
\hline 30 a 39 & 7 & 50 \\
\hline 40 a 49 & 3 & 21,4 \\
\hline 50 ou mais & 1 & 7,1 \\
\hline \multicolumn{3}{|l|}{ Ocupação } \\
\hline Enfermeiro & 9 & 64,3 \\
\hline Téc de enfermagem & 5 & 35,7 \\
\hline \multicolumn{3}{|l|}{ Tempo de trabalho } \\
\hline 0 a 5 & 11 & 78,6 \\
\hline 6 a 10 & 1 & 7,1 \\
\hline Mais de 10 & 2 & 14,3 \\
\hline \multicolumn{3}{|l|}{ Estado civil } \\
\hline Solteiro & 5 & 35,7 \\
\hline Casado & 9 & 64,3 \\
\hline \multicolumn{3}{|l|}{ Cor } \\
\hline Negros & 9 & 74,2 \\
\hline Não negros & 5 & 35,7 \\
\hline Total & 14 & 100 \\
\hline
\end{tabular}

Fonte: Santos APD, et al., 2019.

\section{Entraves para a assistência de enfermagem no ambiente pré-hospitalar}

As características geográficas e os hábitos da população em adotar certas condutas na cena de atendimento configurou-se como um empecilho para os profissionais que almejam seguir uma conduta pautada em protocolo especifico. Nesse contexto, os profissionais apontaram dificuldades relacionadas ao ambiente como fatores que dificultam o atendimento, sobretudo no que diz respeito ao trânsito e acesso ao local de atendimento.

"Durante essa assistência tem vários fatores que podem interferir, desde o local, à gravidade da ocorrência, a chuva que atrapalha um pouco, os locais de acesso ruim, isso tudo influencia na qualidade da assistência" (ENF 9).

“(...)você tem que tá explicando muito, as vezes o público leigo não entende. As vezes eles partem para a ignorância, muitas vezes eles não entendem que nesse município só tem uma base do SAMU e as vezes a gente demora pra chegar por conta da logística de trânsito (...)" (ENF 3).

No que se refere ao acesso ao local de atendimento, as situações de perigo iniciam no trajeto com o risco de colisão, tendo em vista que a ambulância se desloca em alta velocidade durante a ocorrência (FREITAS KO, et al., 2019). Isso ocorre porque a velocidade do deslocamento é fundamental para o bom prognóstico do paciente. No município de Guanambi, no Estado da Bahia, por exemplo, a ambulância do SAMU gasta em média 7,2 minutos para chegar à ocorrência, atendendo a recomendação de tempo de resposta preconizado de até 10 minutos (ALVES ECO, et al., 2014; O'DWYER G, et al., 2017). 
Tanto as limitações geográficas quanto a relação com a população também foram apontadas como fator de interferência na qualidade da assistência em outros estudos. Como não é possível acabar com as limitações relacionadas à geografia do local de atendimento, acreditamos que tal problemática pode ser atenuada através da organização da assistência, com trabalho coordenado para que a assistência seja rápida, eficaz e segura para a equipe (ALVES ECO, et al., 2014; DANTAS GDV, et al., 2019).

Para isso, prioritariamente o profissional deve assegurar-se que o local de atendimento é seguro para que o mesmo consiga exercer seu atendimento de maneira eficiente e com o mínimo de exposição para a equipe e para a vítima (ALMONDES KM, et al., 2016). A proteção da cena evita que ocorra novos acidentes enquanto a assistência é prestada.

Outro fator apontado pelos participantes como entrave para o atendimento é a ocorrência de violência ocupacional perpetrada contra a equipe de socorristas.

“(...)[ocorre] agressões físicas e muito mais coisas. Tipo, agressões físicas são uma das maiores, a população às vezes não entende como o SAMU funciona $e$ acaba vindo com agressões, palavras de baixo calão, e outras coisas" (TEC ENF 2).

“(...)eu costumo falar que a equipe sempre está em perigo (...)” (TEC ENF 3).

“(...) você tem que ter muita cautela pra você abordar um paciente em via pública por que tem muitos curiosos. Às vezes as pessoas não estão ali pra ajudar, mas só pra ver o que está acontecendo, com seus celulares, pra tá fotografando, filmando, [ao invés de] se preocupar realmente com a vida da pessoa (...)" (ENF 1).

Nota-se a existência de violência ocupacional no trabalho de profissionais de enfermagem do APH. A integridade física, moral e psicológica dos profissionais do SAMU estão constantemente expostas às mais diversas situações, desde a divulgação de sua imagem através de registros fotográficos por parte da população, até o risco de lesão corporal (ALMONDES KM, et al., 2016). Estes fatores podem interferir diretamente na assistência prestada, uma vez que o profissional divide a atenção do atendimento entre vítima e população.

Apesar de ser outro contexto sociocultural, é importante mencionar que em estudo realizado no Irã, os enfermeiros relatam que quando ocorrem situações semelhantes às supracitadas, os profissionais tentam ignorar as agressões verbais como forma de assegurar a integridade física da equipe, sobretudo em cenas onde existe um grande aglomerado de pessoas ou em áreas desertas (SCMITH S e CHEMIM BF, 2017).

A exposição excessiva predispõe o surgimento de situações que compromete a integridade do profissional durante a execução de seu trabalho, principalmente por tratar-se de um ambiente não controlado (ALMONDES KM, et al., 2016).

Entretanto, a violência ocupacional não se limita ao ambiente pré-hospitalar. Em estudo realizado com profissionais de enfermagem de um pronto atendimento constatou-se que frequentemente esta classe é vítima de casos de violência ocupacional. Os tipos mais comuns referem-se a situações de violência física e verbal (DADASHZADEH A, et al., 2019). Infere-se assim, que a violência está inserida no setor de urgência e emergência, independente de tratar-se de ambiente extra ou intra hospitalar.

Além da violência praticada pelos transeuntes, há também aquelas perpetradas pelos próprios pacientes. Nesse contexto, o risco de agressão à equipe torna-se mais eminente quando o atendimento é realizado em pacientes psiquiátricos, ou em indivíduos que tiveram seu nível de consciência alterado, podendo ser de origem traumática, relacionado ao ambiente, ou pelo fato de ter havido ingestão de bebida alcoólica (FREITAS KO, et al., 2019).

Com isso, é natural que os profissionais sintam-se inseguros durante o atendimento às ocorrências. Ademais, a falta de investimentos para a segurança da equipe intensifica o risco de agressões que estão expostos constantemente, o que mostra que a temática está invisibilizada por parte da gestão (VASCONCELLOS IRR, et al., 2017). 
Chamamos a atenção para o questionamento em torno das fotos e filmagens na cena do acidente. $O$ avanço tecnológico, onde parcela significativa da população passou a ter acesso aos aparelhos celulares, associado às ocorrências de acidente em locais públicos facilitam o registro de vítimas de acidente e a rápida propagação nos meios digitais, podendo assim gerar desconforto e exposição dos envolvidos na cena (ALMONDES KM, et al., 2016).

Parte dessas dificuldades está associada ao pouco conhecimento da população acerca do papel do SAMU. Acredita-se que apesar de estar presente em todo o território nacional, a função do SAMU ainda é desconhecida por grande parcela da população, fazendo com que muitas pessoas questionem, opinem e intervenham erroneamente durante $o$ atendimento. Ademais, a população leiga acaba realizando manobras que muitas vezes mostram-se equivocadas, o que complica a assistência prestada. Tais fatores podem ser identificados nas falas de ENF2 e ENF3.

“(...) antes de chegar no local de atendimento, antes de chegar a ambulância, geralmente o público leigo tenta fazer alguma manobra (...), algum procedimeno $e$ que a depender do que for feito pode está atrapalhando, pode está piorando a situação do paciente (...) (ENF 2).

“(...) Essa dificuldade está mais relacionada com a falta de conhecimento desse púbico leigo com a função real que do SAMU 192. A população não é bem educada nesse sentido, de orientar qual é a nossa função, aí tem uma certa interferência negativa do público leigo na nossa assistência na rua." (ENF 3).

Percebe-se que parcela da população não compreende a função do SAMU e, a partir do desejo concreto de ajudar, acabam realizando manobras que além de dificultar o atendimento da equipe especializada, pode acabar provocando o agravamento do quadro do paciente.

Essa problemática não se limita à esse estudo. Em pesquisa realizada na Inglaterra que avaliou a presença de transeuntes em APH, foi observado que grande parte das cenas onde teve intervenção direta dessas pessoas na tentativa de realizar manobra de primeiros socorros, houve óbito das vítimas (GARÇOM TL e PUPULIM JSL, 2017).

Isso faz com que, quando existe grande quantidade de pessoas no local da cena, os riscos extrapolem a segurança da equipe, uma vez que pode comprometer o estado de saúde do paciente através da manipulação incorreta das vítimas antes da chegada o socorro.

Destaca-se o fato de não ter sido mencionado pelos participantes outros entraves característicos para a assistência como o alto índice de trotes, acionamento do SAMU em situações que deveria ser resolvida na atenção básica, além de questões gerenciais como os recursos materiais e humanos (OLIVER GJ, et al., 2017).

Tal reflexão se ampara em outros estudos que evidenciaram que muitas pessoas acionam a ambulância para situações que não se caracterizam como urgência ou emergência, comprometendo a utilização do serviço por outro usuário com maior necessidade. Já os trotes fazem com que a equipe aumente os gastos dos serviços, além de elevar o risco de atrasos para a chegada nas cenas onde os acidentes tenham sido reais (OLIVER GJ, et al., 2017).

Ao não discutir questões gerenciais como os recursos escassos, percebemos que o local de fala dos entrevistados se limitou exclusivamente a questões assistenciais. Entretanto, acreditamos que a superação das dificuldades está também atrelada à gestão dos serviços, uma vez que cabe ao gestor assegurar que o serviço prestado à população seja de qualidade (GARÇOM TL e PUPULIM JSL, 2017).

\section{A educação como instrumento de melhoria da assistência prestada no atendimento pré hospitalar}

Diante da problemática levantada pelos profissionais, emerge a necessidade de construção de alternativas, para melhoria da assistência prestada. Nesse sentido, a equipe de enfermagem do SAMU elencou formas utilizadas para melhorar o atendimento, com destaque para a educação e a comunicação com a comunidade e educação permanente para os profissionais. 
"A estratégia é tentar convencer a população mesmo, é explicar a situação que precisa, é a gente tá fazendo dessa forma por que é necessário fazer, a educação mesmo e o diálogo" (ENF 7).

"Você tem que ser bem comunicativo e bem educado com a população, não maltratar ninguém, por que o pessoal já está ali revoltado. Se você for mal educado, o pessoal acaba revoltando com a gente" (TEC ENF 1).

“(...) a gente tem que buscar treinamento por que esse treinamento é o que vai deixar a gente preparada para essas situações inesperadas, (...) [principalmente] através de treinamento e estabelecimento de protocolos para toda a equipe (...)" (ENF 6).

Constata-se que existe grande interesse da equipe em manter uma relação de proximidade com a população, utilizando a comunicação para ter a comunidade como aliada. A comunicação é um instrumento fundamental na área de saúde, principalmente em ambientes não controlados como no APH. A falha na comunicação pode gerar situações inesperadas, frases não compreendidas e falas descontextualizadas, aumentando a tensão na cena de atendimento (SANTOS MC, et al., 2012).

Corroboramos assim com Andrade TF e Silva MMJ (2019) quando pontuam que a educação em saúde com a população surge como espaço de abertura dos canais de comunicação, bem como uma possibilidade de melhoria do serviço, oportunizando que a comunidade faça melhor uso do SAMU.

Esse processo educativo pode sensibilizar a comunidade para o uso correto do serviço, tornando-a também multiplicadora e difusora das informações acerca da função do SAMU. Acredita-se ainda que a sensibilização auxilie na redução de custos, uma vez que a ambulância será poupada de deslocar-se em situações desnecessárias (ANDRADE TF e SILVA MMJ, 2019).

Nesse contexto, acreditamos que a mídia pode ser utilizada de forma estratégica no processo de sensibilização da população acerca dos desafios enfrentados pelo serviço, bem como na forma adequada que a comunidade deve se comportar no momento do atendimento (MORAIS LS, et al., 2015).

A utilização de meios como as emissoras de rádio pode possuir grande efetividade, dada a audiência nos programas diários. Através de um breve comunicado, é possível promover a difusão de informações acerca da função, forma de atendimento e necessidade de respeito à vítima no momento do atendimento, para que reduza o índice de fotos e filmagens sem autorização.

Além da comunidade, o profissional também deve contribuir para superação das dificuldades. Para isso, é importante a implementação de educação permanente, pois devido a complexidade do trabalho exercido, o mesmo deve estar sempre capacitado e atualizado acerca das normas e procedimentos inerentes ao serviço (O'DWYER GJ, et al., 2017), para que seja possível prestar uma assistência ágil e resolutiva.

Implicitamente, nota-se que a superação das dificuldades dos profissionais de enfermagem no APH engloba várias frentes, da micro e macroorganização. Na microorganização, acreditamos ser fundamental ações voltada para educação permanente da equipe de maneira efetiva e periódica, bem como a sensibilização da comunidade em torno função do SAMU para seu uso correto. No aspecto macroorganizacional, é fundamental construir políticas públicas que ofertem melhores condições de trabalho para os profissionais, desde a segurança, até o desenvolvimento de mecanismos de qualificação da assistência prestada.

\section{CONCLUSÃO}

O estudo constatou que as principais dificuldades enfretadas por profissionais de enfermagem no atendimento pré hospitalar estão relacionadas a acessibilidade aos locais de atendimento, segurança da cena e a ocorrência de violência ocupacional contra os profissionais. A superação desses entraves perpassa pela educação permanente da comunidade sobre a função do SAMU e criação de políticas públicas para melhorar as condições de trabalho dos profissionais. 


\section{REFERÊNCIAS}

1. ALMONDES KM, SALES EA, MEIRA MO. Serviço de Psicologia no SAMU: Campo de Atuação em Desenvolvimento. Psicologia: Ciência e Profissão. 2016; 36(2): 449-457.

2. ALVES ECO, et al. Situações enfrentadas pelos enfermeiros no serviço de atendimento pré- hospitalar. Revista Diálogos Acadêmicos. 2014;3(2):1-7.

3. ANDRADE TF, SILVA MMJ. Características dos enfermeiros no atendimento pré-hospitalar: concepções sobre a formação e exercício profissional. Enferm em Foco. 2019; 10 (1):81-86.

4. BARDIN, L. Análise de Conteúdo. 4 ed. Lisboa: Edições 70, 2011.

5. BRASIL. Ministério da Saúde. Portaria no 1.010, de 21 de maio de 2012a. Redefine as diretrizes para a implantação do Serviço de Atendimento Móvel de Urgência (SAMU 192) e sua Central de Regulação das Urgências, componente da Rede de Atenção às Urgências. Brasília: Ministério da Saúde, 2012.

6. Ministério da Saúde. Informações de Saúde (TABNET): Estatísticas Vitais. Sistema de Informações de Mortalidade (SIM). Brasília: Ministério da Saúde, 2018. Disponível em: < http://tabnet.datasus.gov.br/cgi/tabcgi.exe?sim/cnv/ext10uf.def>. Acesso em 02 de Abr. 2018.

7. Ciconet RM. Tempo resposta de um serviço de atendimento móvel de urgência [tese]. Porto Alegre (RS): Escola de Enfermagem, Universidade Federal do Rio Grande do Sul; 2015, 124p.

8. DADASHZADEH A, et al. Iranian pre-hospital emergency care nurses' strategies to manage workplace violence: A descriptive qualitative study. J Nurs Manag. 2019; 27(6):1190-1199.

9. DANTAS GDV, et al. Perfil dos acidentes motociclísticos atendidos pelo Serviço de Atendimento Móvel de Urgência nos anos de 2014 e 2015 em município baiano. J. res.: fundam. care. 2019; 11(4):984-991.

10. FREITAS KO, et al., Atendimento a Saúde por Bombeiros: Dificuldades Encontradas Que Implicam na Assistência a População. Rev Fund Care Online. 2019; 11(n. esp):317-323.

11. GARÇOM TL, PUPULIM JSL. Qualidade do atendimento pré - hospitalar móvel de urgência na perspectiva dos profissionais. Cienc Cuid Saude. 2017 16(4).

12. LIMA ALP, et al. Assistência de enfermagem no atendimento pré-hospitalar, In: International nursing congress, 2017, Aracaju. Anais. Aracajú: UNIT, 2017.

13. LOPES SLB, FERNANDES RJ. Uma breve revisão do atendimento médico pré-hospitalar. Medicina, Ribeirão Preto. 1999; 32(4):381-387.

14. LUCHTEMBERG MN, PIRESS DEP. O que pensam os enfermeiros do SAMU sobre o seu processo de trabalho. Cogitare enferm. 2015; 20(3): 457-466.

15. MACHADO MH, et al. Caracteristicas gerais da enfermagem: o perfil sócio demográfico. Enferm em Foco. 2015; 6 (1/4): 11-17.

16. MATA KSS, et al. Entraves no atendimento pré-hospitalar do samu: percepção dos enfermeiros. Rev enferm UFPE. 2018; 12(8):2137-45.

17. MINAYO MCS. O desafio do conhecimento: pesquisa qualitativa em saúde. 11 ed. São Paulo: Hucitec; 2010, 73p.

18. MORAIS LS, et al. Operacionalização do serviço móvel de urgência em unidades regionalizadas. Rev enferm UFPE. 2015; 9(Supl. 9):9945-56.

19. NATIONAL ASSOCIATION OF EMERGENCY MEDICAL TECHNICIANS. PHTLS: atendimento pré-hospitalar ao traumatizado. 8. ed. Burlington: Jones \& Bartlett Learning, 2017.

20. O'DWYER G, et al. O processo de implantação do Serviço de Atendimento Móvel de Urgência no Brasil: estratégias de ação e dimensões estruturais. Cad. Saúde Pública. 2017; 33 (7): e00043716.

21. OLIVER GJ, et al. Are prehospital deaths from trauma and accidental injury preventable? A direct historical comparison to assess what has changed in two decades. Injury, Int. J. Care Injured. 2017; 48(5): 978-984.

22. SANTOS MC, et al. O processo comunicativo no serviço de atendimento móvel de urgência (samu- 192). Rev. Gaúcha Enferm. 2012; 33(1):69-76.

23. SANTANA JCB, et al. Perfil dos técnicos em enfermagem de um serviço de atendimento pré-hospitalar. Rev. Enfermagem Revista. 2015; 18(1):1-12.

24. SANTOS MC, et al. O processo comunicativo no serviço de atendimento móvel de urgência (samu- 192). Rev. Gaúcha Enferm. 2012; 33(1):69-76.

25. SCMITH S; CHEMIM BF. A violação dos direitos de personalidade de vítimas de acidentes de trânsito pela exposição de imagens. Revista Destaques Acadêmicos. 2017; 9(2):163-184.

26. VASCONCELLOS IRR, et al. Violência ocupacional sofrida pelos profissionais de enfermagem do serviço de pronto atendimento hospitalar. Revista Gaúcha de Enfermagem. 2012; 33(2): 167-175, 2012. 